\section{Systemic lupus erythematosus complicated by diffuse alveolar haemorrhage: risk factors, therapy and survival}

\author{
Nayef M Kazzaz, Patrick Coit, Emily E Lewis, W Joseph McCune, Amr H Sawalha, \\ Jason S Knight
}

To cite: Kazzaz NM, Coit $P$, Lewis EE, et al. Systemic lupus erythematosus complicated by diffuse alveolar haemorrhage: risk factors, therapy and survival. Lupus Science \& Medicine 2015;2:e000117.

doi:10.1136/lupus-2015000117

Received 17 July 2015 Revised 14 August 2015 Accepted 28 August 2015

\section{ABSTRACT}

Objectives: While diffuse alveolar haemorrhage (DAH) is recognised as a life-threatening complication of systemic lupus erythematosus (SLE), little is known about its risk factors and response to treatment. We describe 22 cases of DAH in a US lupus cohort of approximately 1000 patients, and compare them to 66 controls from the same outpatient cohort.

Methods: We captured variables pertaining to diagnoses of SLE and secondary antiphospholipid syndrome (APS), and analysed them by univariate testing. Those variables with $p$ values $<0.05$ were then further considered in a multivariate model. KaplanMeier curves were constructed for each group, and survival was analysed by Log-rank test.

Results: Of the 22 patients with DAH, $59 \%$ were diagnosed with DAH within 5 years of lupus diagnosis. By univariate testing, several manifestations of SLE and APS were more common in patients with DAH, including history of thrombocytopenia, cardiac valve disease, low C3, leucopenia, neuropsychiatric features, haemolysis, arterial thrombosis, lupus anticoagulant, secondary APS and low C4. On multivariate analysis, history of thrombocytopenia and low C3 were maintained as independent risk factors. Importantly, only two patients had platelet counts $<50000 / \mu \mathrm{L}$ at the time of the DAH episode, arguing that DAH was not simply a haemorrhagic complication of thrombocytopenia. All patients were treated with increased immunosuppression, including various combinations of corticosteroids, plasmapheresis, cyclophosphamide, rituximab and mycophenolate mofetil. Notably, all patients in the cohort survived their initial episode of DAH. While the patients with DAH did well in the short-term, their long-term survival was significantly worse than controls. Several of the deaths were attributable to thrombotic complications after recovering from $\mathrm{DAH}$.

Conclusions: To the best of our knowledge, this is the largest case-control study of lupus DAH to date. History of thrombocytopenia was strongly predictive of DAH (OR 40). A number of APS manifestations correlated with DAH by univariate analysis, and deserve further consideration in larger studies.

\section{KEY MESSAGES}

History of thrombocytopenia was a strong predictor of DAH in SLE; however, most patients did not have active thrombocytopenia at the time of DAH.

- Several manifestations of antiphospholipid syndrome correlated with DAH by univariate analysis and deserve further analysis in larger studies.

- While this cohort of DAH patients had very good acute outcomes, they had a long-term survival disadvantage when compared to lupus patients without DAH.

\section{INTRODUCTION}

Diffuse alveolar haemorrhage (DAH) is considered a catastrophic complication of systemic lupus erythematosus (SLE). It can be acute, developing over hours, or subacute, emerging over several days. ${ }^{1}$ Depending on the study, DAH has been reported to complicate $2-5 \%$ of all cases of SLE. ${ }^{2}$ DAH is typically defined by the presence of three major components: (1) signs (bronchoscopy with bloody return) or symptoms (dyspnoea, cough, haemoptysis) of pulmonary haemorrhage, (2) new drop in haemoglobin (typically $1.5-2 \mathrm{~g} / \mathrm{dL}$ ) and (3) new, diffuse infiltrates on chest imaging. ${ }^{4-7}$ Of note, most studies have found dyspnoea to be a far more common presenting symptom than haemoptysis, occurring in $74-100 \%$ of patients, ${ }^{1}{ }^{6-12}$ as compared to $30-100 \%$ for haemoptysis. $\begin{array}{llllll} & 5 & 6 & 8-11 & 13 \\ \text { Lupus nephritis has }\end{array}$ frequently been linked to DAH, with evidence of active kidney disease in as many as $64-100 \%$ of patients with lupus DAH. ${ }^{1}$ 5-8 $1011 \quad 14 \quad 15$ Hypocomplementemia ${ }^{5-7} 11$ and cytopenias $^{5} 7^{8}$ are other common DAHassociated features.

Regarding pathogenesis, lupus DAH is classically reported as a neutrophilic capillaritis 
with destruction of alveolar septae and infiltration of haemosiderin-laden macrophages. ${ }^{14} 1617$ Some studies have additionally shown granular immune complex deposition in the alveolar septae, ${ }^{1416} 17$ while a role for B cells has been suggested by murine models. ${ }^{18}$ Acute DAH mortality in SLE has ranged from $0 \%$ to $62 \%,{ }^{1}{ }^{5-}$ 11131517 with mortality mainly attributable to respiratory complications, ${ }^{14}$ thrombotic disease ${ }^{15}$ and central nervous system disease. ${ }^{13}$ As with most organ-threatening manifestations of SLE, cyclophosphamide has been the mainstay of therapy for DAH, ${ }^{1}{ }^{14}$ albeit in association with poor outcomes, which probably reflect its preferential use in the most critically ill patients. ${ }^{1}$ Other reported therapies include plasmapheresis, ${ }^{19}{ }^{20}$ extracorporeal membrane oxygenation (ECMO), ${ }^{21}{ }^{22}$ rituximab, ${ }^{23-30}$ mycophenolate mofetil, ${ }^{31}$ recombinant factor VII $^{32} 33$ and stem cell transplantation. ${ }^{34}$ The evidence to support any particular therapy is not strong.

Some, but not all, studies have also hinted at a link between antiphospholipid syndrome (APS) and DAH. In lupus DAH, anticardiolipin antibody prevalence has ranged from $12 \%$ to $25 \%,{ }^{689} 11$ with prevalence of the syndrome itself at $13.6 \%{ }^{13}$ In terms of primary APS, several case reports have characterised patients with antiphospholipid antibody-associated or APS-associated $\mathrm{DAH},{ }^{35-38}$ and have found radiological features essentially indistinguishable from those seen in SLE. ${ }^{39}$ Further, at least three primary APS case series have suggested an association between DAH and APS, with some of these cases actually demonstrating biopsy-proven capillaritis. $^{40-42}$

We describe 22 cases of DAH in a US lupus cohort of approximately 1000 patients, and compare them to 66 controls from the same outpatient cohort. To the best of our knowledge, this is the largest case-control study of lupus DAH reported to date.

\section{METHODS}

Patients

The Institutional Review Board of the University of Michigan approved this study, and all patients signed an informed consent form for review of their medical record. The Michigan Lupus Cohort currently includes around 1000 patients who meet the American College of Rheumatology classification criteria for SLE. ${ }^{43}$

We searched the cohort for the terms 'DAH', 'diffuse alveolar haemorrhage' and 'pulmonary haemorrhage'. This search identified 36 patients whose charts were then carefully reviewed. Patients were considered to have DAH if they fulfilled three criteria. The first required the presence of at least one pulmonary sign or symptom, including dyspnoea, cough, haemoptysis, hypoxaemia, intubation or a bronchoscopy positive for bloody return. The second was haemoglobin drop of more than $1.5 \mathrm{~g} / \mathrm{dL}$ over $48 \mathrm{~h}$ (as compared to baseline). The third was abnormal imaging suggestive of a new diffuse infiltrative process, typically either a chest
X-ray or chest CT scan. Twenty-two patients met all three criteria and were considered to have DAH; these were the patients further considered. For each DAH case, three birth-year-matched lupus patients were identified from the same cohort. Therefore, the control group consisted of 66 lupus patients, none of whom had a history of DAH.

\section{Statistical analysis}

Univariate conditional logistic regression was used to evaluate the relationship between DAH and the following predictor variables: sex (male, female), ethnicity (white, black, other), malar rash, the presence of any lupus rash, oral ulcers, alopecia, arthritis, serositis, nephritis, neuropsychological disturbance, Raynaud's phenomenon, leucopenia, haemolysis, thrombocytopenia, low C3, low C4, antinuclear antibodies, anti-dsDNA (double-stranded DNA), anti-Ro (Sjögren's syndrome A, SSA), anti-La (Sjögren's syndrome B, SSB), anti-Sm antibodies, antiribonucleoprotein (RNP), APS, arterial thrombosis, venous thrombosis, pregnancy loss, cardiac valve involvement, livedo reticularis, seizures, anti- $\beta_{2}$ GPI ( $\beta-2$ glycoprotein I) IgG, anti- $\beta_{2} G P I$ IgA, anti- $\beta_{2}$ GPI IgM, anti-cardiolipin IgG, anti-cardiolipin $\operatorname{IgM}$ and lupus anticoagulant.

Predictor variables were ranked based on the $p$ value for the likelihood ratio $\chi^{2}$ test, as well as the OR. A multivariate conditional logistic regression model was built in a stepwise manner. Starting with the highest ranking variable, the next highest ranked variable was added to the equation. The Akaike information criterion (AIC) of the reduced and expanded models and the Wald $p$ values of the predictor variables were compared. A variable was retained in the model if the AIC of the larger model was less than the AIC of the previous model and each variable had a $p$ value $<0.05$. If the variable was rejected, the next ranking variable was introduced and the model was retested. Conditional logistical regression statistical analysis was conducted using SAS V.9.4.

For survival analysis, Kaplan-Meier curves were constructed for each group (DAH vs control), and survival was compared by Log-rank test.

\section{RESULTS}

\section{DAH in SLE}

We identified 22 cases of DAH in our lupus cohort, all of whom fulfilled the three criteria outlined above. Average ages at lupus and DAH diagnoses were 30 and 37 years old, respectively. DAH most commonly presented within 5 years of lupus diagnosis (figure 1). Most patients identified themselves as Caucasian or white $(16 / 22,73 \%)$. Two patients identified themselves as African-American or black, with the remaining patients identifying as Hispanic, Arab or Asian; the race of a single patient could not be determined from the medical record. Interestingly, and similar to prior studies, ${ }^{1}{ }^{5} 68-1113$ only $27 \%$ of patients had haemoptysis 


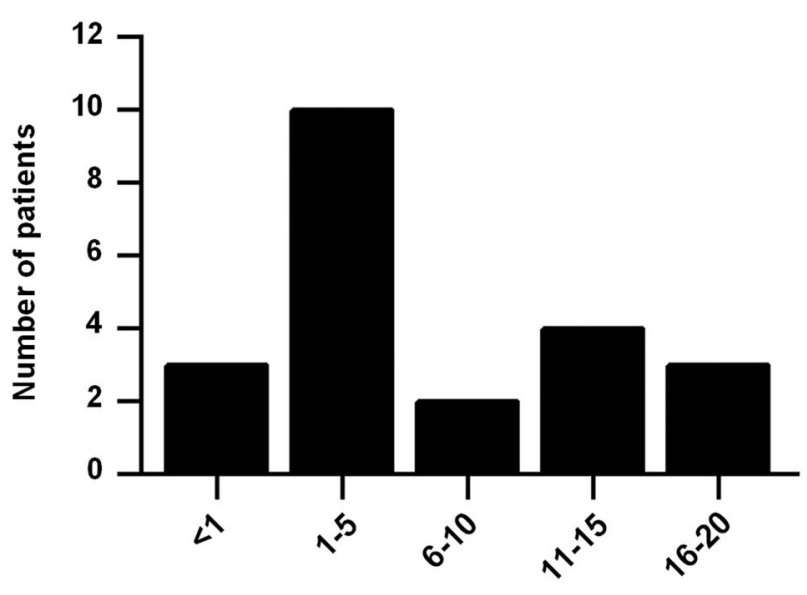

Years (lupus diagnosis to DAH)

Figure 1 Interval between lupus diagnosis and first episode of diffuse alveolar haemorrhage (DAH).

as a presenting symptom (table 1). Not surprisingly, DAH was often associated with worsening hypocomplementemia from baseline $(75 \%)$, as well as increased anti-dsDNA levels from baseline $(56 \%)$. Eight patients $(36 \%)$ were diagnosed with nephritis concurrent to their DAH episode; four of these patients were new diagnoses, and four cases represented reactivation of dormant disease. Average systemic lupus erythematosus disease activity index (SLEDAI)-2K score at presentation was $11.4 .^{44}$

\begin{tabular}{|c|c|c|}
\hline Active features & Frequency & Percentage \\
\hline Dyspnoea & $14 / 22$ & (64) \\
\hline Hypoxaemia* & $12 / 22$ & (55) \\
\hline Cough (no haemoptysis) & $9 / 22$ & (41) \\
\hline Haemoptysis & $6 / 22$ & (27) \\
\hline \multicolumn{3}{|l|}{$\mathrm{BAL}$} \\
\hline Bloody return & $8 / 14$ & (57) \\
\hline Haemosiderin-laden $М \Phi$ & $2 / 14$ & (14) \\
\hline SLEDAI-2K score $†$ & $11.4 \pm 7.3$ & \\
\hline Any lupus rash & $5 / 22$ & (23) \\
\hline Arthritis & $4 / 22$ & (23) \\
\hline Serositis & $7 / 22$ & (32) \\
\hline Nephritis & $8 / 22$ & (36) \\
\hline Neuropsychiatric & $2 / 22$ & (9) \\
\hline Leucopenia & $5 / 21$ & (23) \\
\hline Haemolytic anaemia & $2 / 22$ & (9) \\
\hline Thrombocytopenia & $5 / 22$ & (23) \\
\hline Raynaud's & $2 / 22$ & (9) \\
\hline Hypocomplementemia & $15 / 20$ & (75) \\
\hline Down from baseline & $12 / 16$ & (75) \\
\hline Elevated anti-dsDNA & $14 / 18$ & (78) \\
\hline Up from baseline & $9 / 16$ & (56) \\
\hline \multicolumn{3}{|c|}{$\begin{array}{l}\text { *Nine patients required intubation. } \\
\text { †Mean and SD are presented. } \\
\text { BAL, bronchoalveolar lavage; dsDNA, double-stranded DNA; MФ, } \\
\text { macrophage; SLEDAI, systemic lupus erythematosus disease } \\
\text { activity index } 2000 \text {. }\end{array}$} \\
\hline
\end{tabular}

All DAH diagnoses were made in hospitalised patients. $\mathrm{DAH}$ was the primary indication for admission in 17 of 22 cases $(77 \%)$, while the remainder developed $\mathrm{DAH}$ during hospitalisation for a different indication. Five patients were reportedly on warfarin at the time of the DAH episode, although only one had an elevated international normalised ration (INR) (3.5) at the time of diagnosis; the other four were significantly subtherapeutic (INR 1.1 or lower). Five pulmonary embolism (PE) protocol CT scans were obtained as part of a DAH work up, and all five were negative for PE. Active thrombocytopenia (platelets $<100000 / \mu \mathrm{L}$ ) was present in 5 of 22 patients $(23 \%)$, although only 2 of these patients had severe thrombocytopenia (platelets $<50000 / \mu \mathrm{L}$ ). Eight patients had blood smears ordered to specifically evaluate for microangiopathy, and all eight were negative (including for the two patients who clinically had active haemolysis). Similar to other case series, ${ }^{14512}$ a variety of treatments were employed (table 2). Highlighting a trend in the approach to therapy, all patients treated with rituximab $(3 / 3)$ and $80 \%$ of patients treated with mycophenolate mofetil (4/5) presented after 2007.

\section{Analysis of DAH-associated features}

By univariate analysis, history of several lupus-associated and APS-associated features were more common in patients with DAH than controls (table 3). However, when a multivariate model was constructed, only two features-history of thrombocytopenia $(\mathrm{OR}=36)$ and history of low complement C3 (OR=19)-were maintained as independent risk factors.

\section{Mortality and recurrence}

Although prior studies have shown significant acute mortality from DAH episodes in SLE, this was not apparent in our study, regardless of which treatment was employed. Notably, all 22 patients survived their DAH episode and were discharged from the hospital. Nevertheless, long-term mortality in our DAH cohort was significantly higher when compared to the control

Table 2 Treatment of the first DAH episode

\begin{tabular}{lll}
\hline Treatment & $\begin{array}{l}\text { Frequency } \\
(\mathbf{n = 2 2 )}\end{array}$ & Percentage \\
\hline Pulse methylprednisolone & 10 & 45 \\
Prednisone 1 mg/kg/day & 4 & 18 \\
Increased prednisone & 7 & 32 \\
Intravenous & 3 & 14 \\
immunoglobulin & & \\
Plasmapheresis & 4 & 18 \\
Cyclophosphamide (any) & 9 & 41 \\
Rituximab (any) & 3 & 14 \\
Cyclophosphamide & 2 & 9 \\
+rituximab & & \\
Mycophenolate mofetil & 5 & 23 \\
\hline DAH, diffuse alveolar haemorrhage.
\end{tabular}


Table 3 Comparison between DAH and control groups (univariate analysis)

\begin{tabular}{|c|c|c|c|c|}
\hline Historical features & $\mathrm{DAH}(\mathrm{n}=22)$ & Control $(n=66)$ & OR (CI) & p Value \\
\hline Malar rash & $8 / 22$ & $33 / 66$ & 0.61 (0.24 to 1.56$)$ & 0.3 \\
\hline Any lupus rash & $17 / 22$ & $44 / 66$ & $1.65(0.56$ to 4.93$)$ & 0.35 \\
\hline Oral ulcers & $6 / 22$ & $16 / 66$ & $1.16(0.41$ to 3.28$)$ & 0.79 \\
\hline Alopecia & $5 / 22$ & $10 / 66$ & $1.6(0.50$ to 5.09$)$ & 0.44 \\
\hline Arthritis & $19 / 22$ & $63 / 66$ & $0.18(0.02$ to 1.82$)$ & 0.11 \\
\hline Serositis & $14 / 22$ & $33 / 66$ & 2.07 (0.67 to 6.36$)$ & 0.20 \\
\hline Nephritis & $14 / 22$ & $28 / 66$ & 2.52 (0.89 to 7.16$)$ & 0.07 \\
\hline Neuropsychiatric & $10 / 22$ & $9 / 66$ & 9.20 (1.94 to 43.59$)$ & 0.001 \\
\hline Raynaud's & $5 / 22$ & $24 / 66$ & $0.54(0.18$ to 1.59$)$ & 0.24 \\
\hline Haemolysis & $12 / 22$ & $10 / 66$ & 5.85 (2.01 to 17.03$)$ & 0.001 \\
\hline Thrombocytopenia* & $19 / 22$ & $10 / 66$ & 43.2 (5.72 to 326.40$)$ & $<0.0001$ \\
\hline Leucopenia & $21 / 22$ & $39 / 66$ & 13.43 (1.74 to 103.79$)$ & 0.0004 \\
\hline Low C3* & $20 / 22$ & $29 / 66$ & 19.26 (2.49 to 148.72$)$ & $<0.0001$ \\
\hline Low C4 & $16 / 22$ & $31 / 66$ & $3.32(1.05$ to 10.54$)$ & 0.03 \\
\hline ANA & $21 / 22$ & $58 / 64$ & 2.27 (0.26 to 20.06$)$ & 0.43 \\
\hline Anti-dsDNA & $18 / 22$ & $41 / 65$ & 2.45 (0.77 to 7.77$)$ & 0.11 \\
\hline Anti-SSA & $7 / 21$ & $27 / 64$ & 0.65 (0.21 to 1.98$)$ & 0.44 \\
\hline Anti-SSB & $3 / 21$ & $13 / 63$ & 0.70 (0.19 to 2.64$)$ & 0.6 \\
\hline Anti-Sm & $8 / 21$ & $16 / 63$ & 2.59 (0.79 to 8.45$)$ & 0.11 \\
\hline Anti-RNP & $8 / 21$ & $22 / 63$ & $1.23(0.44$ to 3.44$)$ & 0.69 \\
\hline Arterial thrombosis & $7 / 22$ & $4 / 66$ & 6.42 (1.65 to 25.09$)$ & 0.01 \\
\hline Venous thrombosis & $7 / 22$ & $10 / 66$ & $2.62(0.84$ to 8.15$)$ & 0.1 \\
\hline Pregnancy loss & $3 / 18$ & $4 / 60$ & 2.81 (0.56 to 13.96$)$ & 0.22 \\
\hline Cardiac valve disease & $8 / 22$ & $2 / 66$ & 22.09 (2.75 to 177.67$)$ & $<0.0001$ \\
\hline Livedo reticularis & $2 / 22$ & $5 / 66$ & $1.2(0.23$ to 6.19$)$ & 0.83 \\
\hline Seizures & $4 / 22$ & $4 / 66$ & 4.37 (0.77 to 24.92$)$ & 0.09 \\
\hline Anti- $\beta_{2} G P I \lg G$ & $2 / 21$ & $9 / 55$ & $0.59(0.11$ to 3.05$)$ & 0.52 \\
\hline Anti- $\beta_{2}$ GPI lgA & $3 / 21$ & $11 / 55$ & 0.70 (0.18 to 2.80$)$ & 0.61 \\
\hline Anti- $\beta_{2}$ GPI IgM & $1 / 21$ & $3 / 55$ & $1.0(0.10$ to 9.61$)$ & 1 \\
\hline Cardiolipin IgG & $7 / 22$ & $16 / 63$ & 1.35 (0.46 to 3.98$)$ & 0.58 \\
\hline Cardiolipin IgM & $2 / 22$ & $4 / 62$ & 1.39 (0.26 to 7.66$)$ & 0.71 \\
\hline Lupus anticoagulant & $9 / 20$ & $7 / 60$ & 5.36 (1.63 to 17.68$)$ & 0.004 \\
\hline APS & $6 / 22$ & $6 / 66$ & $4.37(1.06$ to 18.11$)$ & 0.04 \\
\hline
\end{tabular}

${ }^{*}$ Maintained as statistically significant risk factors in a multivariate model.

Statistically-significant $p$ values $(p<0.05)$ are presented in bold.

ANA, antinuclear antibody; APS, antiphospholipid syndrome; DAH, diffuse alveolar haemorrhage; dsDNA, double-stranded DNA; RNP, ribonucleoprotein; SSA, Sjögren's syndrome A; SSB, Sjögren's syndrome B; $\beta_{2}$ GPI, $\beta$-2 glycoprotein I.

group (figure 2A). Causes of death in patients with DAH included stroke (2 patients), infectious complications (2), neurological decline (1) and unknown (1). DAH recurred in 6 of 22 patients $(27 \%)$, typically within 6 months of the original presentation (figure 2B).

\section{DISCUSSION}

To the best of our knowledge, we present the largest case-control study of lupus DAH reported to date. There were similarities and differences between our findings and those in the work of others. For example, similar to previous studies, we found that dyspnoea was a more common presentation than haemoptysis. ${ }^{1}$ 4-6 8-13 While active nephritis was often associated with DAH in our study (36\% of patients), the percentage was somewhat lower than what has been described by others. ${ }^{15-8}$ 10111415 In comparison to the other large case series from Asia and South America, ${ }^{4-7}$ we describe a US lupus population that is predominantly of European descent. This difference in ethnicity may have contributed to the lower percentage of nephritis. ${ }^{45-47}$ It should also be pointed out that kidney biopsies were typically only obtained if the clinician felt it would change clinical management. Given the degree of serological activity that was present in these patients (75\% had decreased complement levels from baseline), it is reasonable to assume that several additional patients had early or subclinical nephritis that may have been effectively treated by the potent immunosuppression employed for $\mathrm{DAH}^{48}{ }^{49}$

Also notable is that all 22 patients survived their first DAH episode; further, although 6 patients had recurrence, none resulted in death. In contrast, prior studies have shown acute mortality as high as 62\%. ${ }^{1}$ 5-9 111317 Ours is a predominantly outpatient cohort, with the majority of patients recruited and enrolled during a visit to outpatient clinic. These patients were carefully followed and monitored, and it is possible that this 


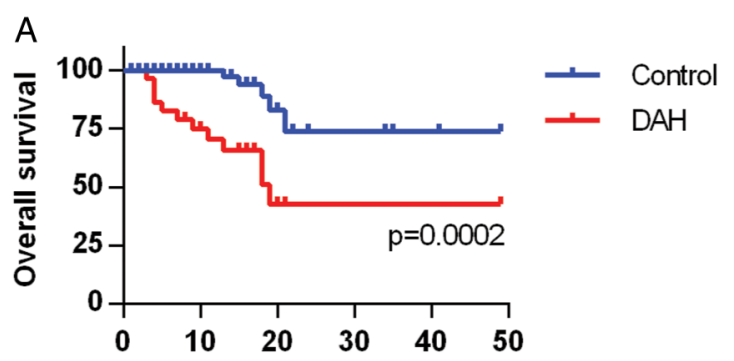

Time from lupus diagnosis (years)

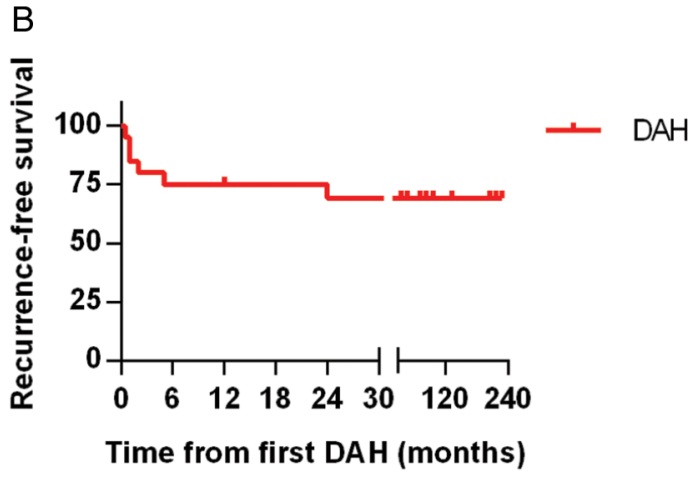

Figure 2 Overall and recurrence-free survival. (A) Kaplan-Meier curve comparing mortality of the lupus diffuse alveolar haemorrhage (DAH) group and the lupus controls. Lupus diagnosis is the point of reference and is set as time zero. (B) Kaplan-Meier curve demonstrating DAH recurrence-free survival in the lupus DAH group. Notably, all but one recurrence was within 6 months of the original episode.

attention resulted in earlier diagnosis as well as more aggressive treatment. It is also notable that the majority of our patients $(14 / 22,64 \%)$ were diagnosed after 2007 ; it is therefore conceivable that newer therapeutic agents such as mycophenolate mofetil and rituximab

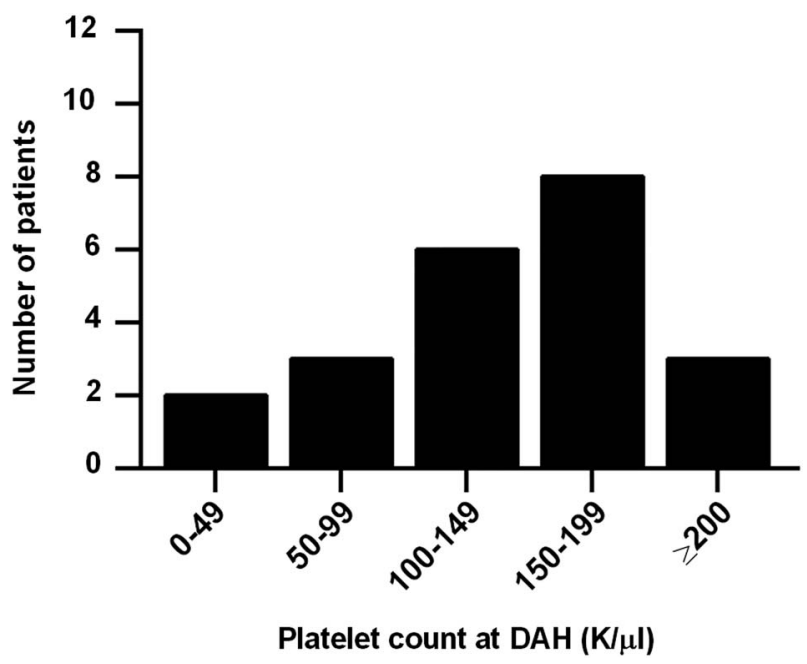

Figure 3 Platelet count at the time of first episode of diffuse alveolar haemorrhage (DAH). While only five patients had a platelet count $<100000 / \mu \mathrm{L}$ at the time of the DAH episode, 19 of 22 had such a count as part of their lupus history. contributed to better outcomes and more durable induction of remission. Finally, it should again be pointed out that this is the first time a lupus population predominantly of European descent has been considered in a large DAH case series; as such, it is possible that this is a population predisposed to better outcomes. $^{115}$

There are no randomised clinical trials to inform our management of patients with lupus DAH. Retrospective studies have shown a survival disadvantage with cyclophosphamide, although it has been speculated that this is the result of cyclophosphamide's preferential use in the most ill patients. ${ }^{1}{ }^{4}$ Such studies have also been unable to show a clear survival benefit of either intravenous immunoglobulin or plasmapheresis in lupus DAH, although these treatments are commonly employed in severe cases (including at our centre). ${ }^{15} 12$ Rituximab is another emerging treatment, although again without either retrospective or prospective data to support its use. ${ }^{4}$ In the absence of clear guidance from the literature, the approach to treatment remains individualised.

Of interest, history of both leucopenia and thrombocytopenia were strongly associated with DAH, with history of thrombocytopenia, in particular, being the strongest individual predictor of DAH in our study. In fact, after multivariate modelling, all risk was explained by just two factors: history of thrombocytopenia and history of low complement C3. We initially considered the possibility that DAH might be explained by the bleeding diathesis of thrombocytopenia itself, but this seems unlikely as only five patients presented with platelet counts below $100000 / \mu \mathrm{L}$ and only two with platelets $<50000 / \mu \mathrm{L}$ (figure 3). For the remainder, the thrombocytopenia $(<100000 / \mu \mathrm{L})$ was part of their medical history, but not active at the time of DAH. It should also be pointed out that thrombocytopenia, similar to nephritis and neuropsychiatric disease, is a well-known predictor of worse prognosis in lupus. ${ }^{50-53}$ Platelets are known mediators of inflammation, for example by engaging neutrophils, ${ }^{54}{ }^{55}$ and may be depleted systemically as they concentrate at sites of local inflammation. It has also been suggested that there are immunological difference between the antiplatelet immune response in lupus, as compared to idiopathic immune thrombocytopenia, ${ }^{56}$ although it is unclear what implications this may have for other aspects and manifestations of SLE.

Although thrombocytopenia ultimately dominated the multivariate model, we did note that a number of APS features, including lupus anticoagulant, arterial thrombosis, valve disease and the syndrome itself, were associated with DAH by univariate analysis. Since APS can also drive thrombocytopenia, ${ }^{57}$ these relationships are complicated and will need to be clarified in larger cohorts that ideally also include patients with primary APS. It is noteworthy that in our series, two of the patients with DAH ultimately died of thrombotic strokes, while a third had a progressive neurological decline related to refractory seizures that may have been driven 
by APS. While the relationship between lupus DAH and APS has not been thoroughly explored in the literature, there are many reports and series describing DAH as a complication of primary APS ${ }^{35-38}$ Again, given the likely complex pathophysiology, these relationships will need to be tracked in larger studies.

Our study has several limitations. The rarity and acuity of DAH limit our ability to obtain prospective data, although this would obviously be the preferred methodology. As highlighted above, this is also a cohort that was primarily established in our outpatient clinics. It is therefore possible that we missed patients, especially those with poor and acute outcomes, who received their outpatient care elsewhere and were transferred to our centre for inpatient management. These data should therefore be interpreted in that light. Finally, because the acute outcomes were so good, we were not able to comment on the response to different types of treatments, which had been a goal when we devised the study.

In summary, using a case-control methodology, we showed a significant association between history of thrombocytopenia and lupus DAH. In fact, an amazing 19 of 22 patients $(86 \%)$ had a history of platelets $<100000 / \mu \mathrm{L}$ at some point during their lupus course, and we would suggest that DAH should be featured prominently in the differential diagnosis when such patients present with pulmonary complaints. This is especially true as haemoptysis is only present in about one-in-four presentations, and therefore the index of suspicion must be high to establish the diagnosis of DAH. Further, although thrombocytopenia dominated the multivariate model, our univariate analysis suggests that secondary APS (similar to primary APS) may be a risk factor for DAH. While we await with interest larger multicentre considerations of this rare, but dangerous, lupus complication, we were encouraged to find that a carefully monitored cohort can fair well with DAH, especially acutely.

Funding JSK was supported by NIH K08AR066569 and a career development award from the Burroughs Wellcome Fund. WJM and the Michigan Lupus Cohort were supported by the Mary Piazza Lupus Research Fund and the Michael and Marcia Klein Lupus Research Fund.

Competing interests None declared.

Ethics approval University of Michigan Institutional Review Board.

Provenance and peer review Not commissioned; externally peer reviewed.

Data sharing statement No additional data are available.

Open Access This is an Open Access article distributed in accordance with the terms of the Creative Commons Attribution (CC BY 4.0) license, which permits others to distribute, remix, adapt and build upon this work, for commercial use, provided the original work is properly cited. See: http:// creativecommons.org/licenses/by/4.0/

\section{REFERENCES}

1. Zamora MR, Warner ML, Tuder R, et al. Diffuse alveolar hemorrhage and systemic lupus erythematosus. Clinical presentation, histology, survival, and outcome. Medicine (Baltimore) 1997;76:192-202.
2. Pego-Reigosa JM, Medeiros DA, Isenberg DA. Respiratory manifestations of systemic lupus erythematosus: old and new concepts. Best Pract Res Clin Rheumatol 2009;23:469-80.

3. Torre $\mathrm{O}$, Harari S. Pleural and pulmonary involvement in systemic lupus erythematosus. Presse Med 2011;40(1 Pt 2):e19-29.

4. Martinez-Martinez MU, Sturbaum AK, Alcocer-Varela J, et al. Factors associated with mortality and infections in patients with systemic lupus erythematosus with diffuse alveolar hemorrhage. $J$ Rheumatol 2014;41:1656-61.

5. Kwok SK, Moon SJ, Ju JH, et al. Diffuse alveolar hemorrhage in systemic lupus erythematosus: risk factors and clinical outcome: results from affiliated hospitals of Catholic University of Korea. Lupus 2011;20:102-7.

6. Chang MY, Fang JT, Chen YC, et al. Diffuse alveolar hemorrhage in systemic lupus erythematosus: a single center retrospective study in Taiwan. Renal Failure 2002;24:791-802.

7. Cañas C, Tobón GJ, Granados M, et al. Diffuse alveolar hemorrhage in Colombian patients with systemic lupus erythematosus. Clin Rheumatol 2007;26:1947-9.

8. Martínez-Martínez MU, Abud-Mendoza C. Predictors of mortality in diffuse alveolar haemorrhage associated with systemic lupus erythematosus. Lupus 2011;20:568-74.

9. Koh WH, Thumboo J, Boey ML. Pulmonary haemorrhage in oriental patients with systemic lupus erythematosus. Lupus 1997;6:713-16.

10. Santos-Ocampo AS, Mandell BF, Fessler BJ. Alveolar hemorrhage in systemic lupus erythematosus: presentation and management. Chest 2000;118:1083-90.

11. Shen $M$, Zeng $X$, Tian X, et al. Diffuse alveolar hemorrhage in systemic lupus erythematosus: a retrospective study in China. Lupus 2010;19:1326-30.

12. Martínez-Martínez MU, Abud-Mendoza C. Diffuse alveolar hemorrhage in patients with systemic lupus erythematosus. Clinical manifestations, treatment, and prognosis. Reumatol Clin 2014;10:248-53.

13. Badsha $\mathrm{H}$, Teh $\mathrm{CL}$, Kong $\mathrm{KO}$, et al. Pulmonary hemorrhage in systemic lupus erythematosus. Semin Arthritis Rheum 2004;33:414-21.

14. Eagen JW, Memoli VA, Roberts JL, et al. Pulmonary hemorrhage in systemic lupus erythematosus. Medicine (Baltimore) 1978;57:545-60.

15. Schwab EP, Schumacher HR, Freundlich B, et al. Pulmonary alveolar hemorrhage in systemic lupus erythematosus. Semin Arthritis Rheum 1993;23:8-15.

16. Fishbein GA, Fishbein MC. Lung vasculitis and alveolar hemorrhage: pathology. Semin Respir Crit Care Med 2011;32:254-63.

17. Barile LA, Jara LJ, Medina-Rodriguez F, et al. Pulmonary hemorrhage in systemic lupus erythematosus. Lupus 1997;6:445-8.

18. Barker TT, Lee PY, Kelly-Scumpia KM, et al. Pathogenic role of B cells in the development of diffuse alveolar hemorrhage induced by pristane. Lab Invest 2011;91:1540-50.

19. Verzegnassi F, Marchetti F, Zennaro F, et al. Prompt efficacy of plasmapheresis in a patient with systemic lupus erythematosus and diffuse alveolar haemorrhage. Clin Exp Rheumatol 2010;28:445-6.

20. Claridge $S$, Das $P$, Dorling $A$, et al. Plasmapheresis as rescue therapy for systemic lupus erthyematosus-associated diffuse alveolar haemorrhage. BMJ Case Rep 2011;2011:pii: bcr0220113893.

21. Patel JJ, Lipchik RJ. Systemic lupus-induced diffuse alveolar hemorrhage treated with extracorporeal membrane oxygenation: a case report and review of the literature. $J$ Intensive Care Med 2014;29:104-9.

22. Pacheco Claudio C, Charbonney E, Durand M, et al. Extracorporeal membrane oxygenation in diffuse alveolar hemorrhage secondary to systemic lupus erythematosus. J Clin Med Res 2014;6:145-8.

23. Nellessen CM, Pöge U, Brensing KA, et al. Diffuse alveolar haemorrhage in a systemic lupus erythematosus patient successfully treated with rituximab: a case report. Nephrol Dial Transplant 2008:23:385-6.

24. Pinto LF, Candia L, Garcia P, et al. Effective treatment of refractory pulmonary hemorrhage with monoclonal anti-CD20 antibody (rituximab). Respiration 2009;78:106-9.

25. Narshi CB, Haider S, Ford CM, et al. Rituximab as early therapy for pulmonary haemorrhage in systemic lupus erythematosus. Rheumatology (Oxford) 2010;49:392-4.

26. Pottier V, Pierrot M, Subra JF, et al. Successful rituximab therapy in a lupus patient with diffuse alveolar haemorrhage. Lupus 2011;20:656-9.

27. Martínez-Martínez MU, Abud-Mendoza C. Recurrent diffuse alveolar haemorrhage in a patient with systemic lupus erythematosus: long-term benefit of rituximab. Lupus 2012;21:1124-7. 
28. Tse JR, Schwab KE, McMahon M, et al. Rituximab: an emerging treatment for recurrent diffuse alveolar hemorrhage in systemic lupus erythematosus. Lupus 2015;24:756-9.

29. Na JO, Chang $\mathrm{SH}$, Seo KH, et al. Successful early rituximab treatment in a case of systemic lupus erythematosus with potentially fatal diffuse alveolar hemorrhage. Respiration 2015;89:62-5.

30. Abud-Mendoza C, Moreno-Valdés R, Cuevas-Orta E, et al. Treating severe systemic lupus erythematosus with rituximab. An open study. Reumatol Clin 2009;5:147-52.

31. Al Rashidi A, Alajmi M, Hegazi MO. Mycophenolate mofetil as a maintenance therapy for lupus-related diffuse alveolar hemorrhage: a case report. Lupus 2011;20:1551-3.

32. Alabed IB. Treatment of diffuse alveolar hemorrhage in systemic lupus erythematosus patient with local pulmonary administration of factor VIla (rFVIla): a case report. Medicine (Baltimore) 2014;93:e72.

33. Esper RC, Estrada IE, de la Torre León T, et al. Treatment of diffuse alveolar hemorrhage secondary to lupus erythematosus with recombinant activated factor VII administered with a jet nebulizer. $J$ Intensive Care 2014;2:47.

34. Liang J, Gu F, Wang $\mathrm{H}$, et al. Mesenchymal stem cell transplantation for diffuse alveolar hemorrhage in SLE. Nat Rev Rheumatol 2010;6:486-9.

35. Howe HS, Boey ML, Fong KY, et al. Pulmonary haemorrhage, pulmonary infarction, and the lupus anticoagulant. Ann Rheum Dis 1988;47:869-72.

36. Asherson RA, Greenblatt MA. Recurrent alveolar hemorrhage and pulmonary capillaritis in the "primary" antiphospholipid syndrome. $J$ Clin Rheumatol 2001;7:30-3.

37. Scheiman Elazary A, Klahr PP, Hershko AY, et al. Rituximab induces resolution of recurrent diffuse alveolar hemorrhage in a patient with primary antiphospholipid antibody syndrome. Lupus 2012;21:438-40.

38. Hillerdal G, Hägg A, Licke G, et al. Intra-alveolar haemorrhage in the anticardiolipin antibody syndrome. Scand J Rheumatol 1991;20:58-62.

39. Nguyen VA, Gotwald T, Prior C, et al. Acute pulmonary edema, capillaritis and alveolar hemorrhage: pulmonary manifestations coexistent in antiphospholipid syndrome and systemic lupus erythematosus? Lupus 2005;14:557-60.

40. Cartin-Ceba R, Peikert T, Ashrani A, et al. Primary antiphospholipid syndrome-associated diffuse alveolar hemorrhage. Arthritis Care Res (Hoboken) 2014:66:301-10.

41. Yachoui R, Sehgal R, Amlani B, et al. Antiphospholipid antibodies-associated diffuse alveolar hemorrhage. Semin Arthritis Rheum 2015;44:652-7.
42. Deane KD, West SG. Antiphospholipid antibodies as a cause of pulmonary capillaritis and diffuse alveolar hemorrhage: a case series and literature review. Semin Arthritis Rheum 2005;35:154-65.

43. Hochberg MC. Updating the American College of Rheumatology revised criteria for the classification of systemic lupus erythematosus. Arthritis Rheum 1997;40:1725

44. Gladman DD, Ibanez D, Urowitz MB. Systemic lupus erythematosus disease activity index 2000. J Rheumatol 2002;29:288-91.

45. Lau CS, Yin G, Mok MY. Ethnic and geographical differences in systemic lupus erythematosus: an overview. Lupus 2006;15:715-19.

46. Gonzalez LA, Toloza SM, McGwin G, Jr, et al. Ethnicity in systemic lupus erythematosus (SLE): its influence on susceptibility and outcomes. Lupus 2013;22:1214-24.

47. Somers EC, Marder W, Cagnoli P, et al. Population-based incidence and prevalence of systemic lupus erythematosus: the Michigan Lupus Epidemiology and Surveillance program. Arthritis Rheumatol 2014;66:369-78.

48. Valente de Almeida R, Rocha de Carvalho JG, de Azevedo VF, et al. Microalbuminuria and renal morphology in the evaluation of subclinical lupus nephritis. Clin Nephrol 1999;52:218-29.

49. Balow JE. Clinical presentation and monitoring of lupus nephritis. Lupus 2005;14:25-30.

50. Fayyaz A, Igoe A, Kurien BT, et al. Haematological manifestations of lupus. Lupus Sci Med 2015;2:e000078.

51. Mok CC, Lee KW, Ho CT, et al. A prospective study of survival and prognostic indicators of systemic lupus erythematosus in a southern Chinese population. Rheumatology (Oxford) 2000;39:399-406.

52. Pistiner M, Wallace DJ, Nessim S, et al. Lupus erythematosus in the 1980s: a survey of 570 patients. Semin Arthritis Rheum 1991;21:55-64.

53. Reveille JD, Bartolucci A, Alarcon GS. Prognosis in systemic lupus erythematosus. Negative impact of increasing age at onset, black race, and thrombocytopenia, as well as causes of death. Arthritis Rheum 1990;33:37-48.

54. Nording HM, Seizer P, Langer HF. Platelets in inflammation and atherogenesis. Front Immunol 2015;6:98.

55. Grommes J, Alard JE, Drechsler M, et al. Disruption of platelet-derived chemokine heteromers prevents neutrophil extravasation in acute lung injury. Am J Respir Crit Care Med 2012;185:628-36.

56. Howe SE, Lynch DM. Platelet antibody binding in systemic lupus erythematosus. J Rheumatol 1987:14:482-6.

57. Drenkard C, Villa AR, Alarcon-Segovia D, et al. Influence of the antiphospholipid syndrome in the survival of patients with systemic lupus erythematosus. J Rheumatol 1994;21:1067-72. 